\title{
Características Biométricas de la Arteria Coroidea Anterior en su Segmento Cisternal
}

\author{
Biometrical Characteristics of Anterior Choroidal Artery in its Cisternal Segment
}

\author{
"Eduardo Mandiola; "Eduardo Alarcón; "Carolina Montero; **Marcos Pulleghini;" \\ ${ }^{* *}$ Mariano del Sol; ${ }^{* * *}$ Enrique Olave $\mathcal{\&}^{* *}$ Juan C. Oñate
}

\begin{abstract}
MANDiOla, E.; AlARCón, E.; MONTERO, C.; PULleghini, M.; DEL SOL, M.; OLAVE, E. \& OÑATE, J. C. Características biométricas de la arteria coroídea anterior en su segmento cisternal. Int. J. Morphol., 30(3):1050-1055, 2012.

RESUMEN: Las implicancias clínicas que tienen los procesos oclusivos de la Arteria Coroidea Anterior (AChA), son de considerable importancia y nos han motivado para efectuar su análisis biométrico incorporando algunos otros parámetros. Para su ejecución, se utilizaron cerebros inyectados con látex coloreado de rojo fijados en solución de formol, provenientes del Servicio Médico Legal, Santiago. Para su observación, se usó lupa luminiscente $4 \mathrm{X}$ y las mediciones efectuadas con Caliper Mitutoyo Digital. Esta arteria se originó de la Carótida Interna (ACI) en un $83,3 \%$ y en un $16,7 \%$ de la Arteria Comunicante Posterior (ACoP ). El calibre medio observado en su origen fue de $0,68 \pm 0,31 \mathrm{~mm}$; en el tercio medio de 0,60 $\pm 0,21 \mathrm{~mm}$; en el punto de entrada en el plexo coroideo de $0,48 \pm 0,23 \mathrm{~mm}$. La longitud media desde el origen de la AChA al plexo coroideo fue de 28,67 $\pm 6,33 \mathrm{~mm}$; la distancia media desde su origen al de la ACoP fue de $6,97 \pm 4,89 \mathrm{~mm}$. El número promedio de ramas perforantes registradas fue de 6 . Nuestros resultados pueden ser una contribución a considerar en la clínica.
\end{abstract}

PALABRAS CLAVE : Arteria coroidea anterior; Biometría; Índice cefálico; Arteria carótida interna.

\section{INTRODUCCIÓN}

Dada la importancia en intervenciones microquirúrgicas y su relación topográfica, la arteria coroídea anterior (AChA) ha sido estudiada por diversos autores clásicos y por anatomistas clínicos.

Autores, en trabajos previos, han hecho notar la complejidad y variabilidad del trayecto de las arterias componentes del círculo arterial cerebral, lo que nos motiva a realizar un trabajo relacionado con la AChA (Mandiola et al., 2006, 2010).

La AChA que se origina frecuentemente de la arteria carótida interna (ACI) es delgada y larga (1 a $1,5 \mathrm{~mm}$ ), se dirige hacia atrás, siguiendo el tracto óptico, rodeando en dirección dorsal la parte más anterior del mesencéfalo, al cual aporta varias ramas.

Sigue su curso cisternal, penetra en el cuerno temporal del ventrículo lateral a través de la fisura coroídea y contribuye a irrigar los plexos coroides junto con las arterias coroideas posteriores medial y lateral, ramas de la parte proximal de la arteria cerebral posterior (ACP) (Testut \& Latarjet, 1964 ).

En su trayecto la AChA proporciona ramas al uncus del parahipocampo, cuerpo amigdalino, cuerpos estriados, sustancia negra, núcleo rojo, fimbria del hipocampo, tracto óptico, radiaciones ópticas y al brazo occipital de la cápsula interna.

Según Truek \& Carpenter (1973), la AChA frecuentemente proviene de la ACI, distal al origen de la arteria comunicante posterior (ACoP), pero puede originarse también de la arteria cerebral media (ACM) o de la ACoP. También hacen notar a la AChA como un vaso muy susceptible a la trombosis, debido a su largo curso subaracnoideo y su calibre pequeño, no mayor a $1 \mathrm{~mm}$.

Autores clásicos de la Anatomía tales como Adachi (1928); OrtsLLorca, (1964), Testut \& Latarjet; Warwick\& Williams (1979); Rouvière \& Delmas (1984) entre otros, mencionan las arterias coroídeas en sus tratados, sin precisar detalles biométricos.

\footnotetext{
* Facultad de Medicina, Universidad Andrés Bello, Santiago, Chile.

** Servicio Médico Legal, Santiago, Chile.

*** Facultad de Medicina, Universidad de La Frontera, Temuco, Chile. Parcialmente financiado por DI-UNAB-02-05/ R
} 
En la literatura clínica hay numerosas referencias al calibre y trayecto de la AChA, así lo describe Wackenheim et al. (1976); Saeki \& Rhoton (1977); Rhoton et al. (1979); Lazorthes et al. (1979); Orlandini et al. (1985); Ferreira \& Braga (1990) y Marinkovic et al. (1999).

Milisavljevic et al. (1986) y Servet et al. (2007) especificaron que las variaciones en el calibre de los vasos, edad y sexo, más factores genéticos son factores a considerar, así como los patrones morfológicos en la interpretación de pruebas arteriográficas. Incluso estos clínicos, han demostrado gran variabilidad en estos vasos y hallazgos en el campo operatorio no descritos en textos.

Nuestra investigación está orientada hacia la variabilidad que se observa cuando se considera, calibre, longitud, origen, sexo y datos bioantropológicos.

Se pretende contribuir al conocimiento cada vez más necesario en la práctica clínica, considerando el alto grado de mestizaje de nuestras poblaciones.

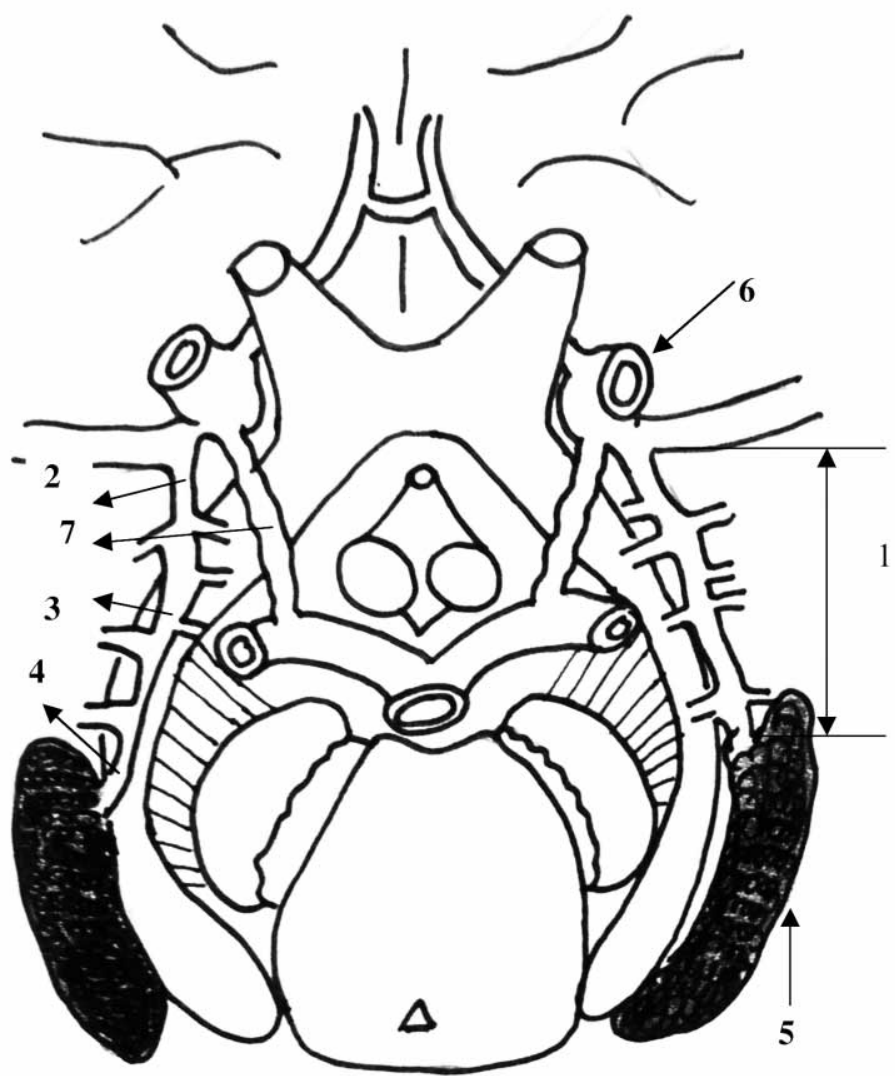

Fig. 1 . Esquema que muestra imagen ventral de encéfalo y conformación del círculo arterial del cerebro. 1. Longitud arteria coroídea anterior (AChA) 2. Calibre origen AChA. 3. Calibre tercio medio AChA. 4. Calibre entrada punto plexal. 5. Plexo coroideo ventrículo lateral. 6. Arteria carótida interna. 7. Arteria comunicante posterior

\section{MATERIAL Y MÉTODO}

Se utilizaron encéfalos de adultos, de ambos sexos, recolectados en el Laboratorio de Anatomía y Servicio Médico Legal de Santiago, con registro antropológico y aportes de nuestro Banco de Datos. Para las mediciones cefálicas utilizamos la Técnica de Martin \& Saller (1957).

Se estudiaron desde el punto de vista biométrico, 32 hemisferios cerebrales, considerando la correlación correspondiente con dimensiones de longitud y calibre de la AChA por segmentos y ramas perforantes de su parte cisternal.

Todas las muestras se fotografiaron con cámara Nikon D40X y los registros en protocolos ad hoc. Las disecciones fueron realizadas bajo lupa 4X . Las mediciones se efectuaron con caliper Mitutoyo digital y con el Programa computacional Carnoy.

El análisis estadístico se realizó mediante los test de student y de Pearson para analizar relaciones entre las variables observadas.

\section{RESULTADOS}

La longitud antero-posterior de la cabeza promedio fue de $175,6 \mathrm{~mm} \pm 14,1 \mathrm{~mm}$, el diámetro biparietal de $139,3 \mathrm{~mm} \pm 22,6 \mathrm{~mm}$; la altura cefálica tuvo un promedio de $130,5 \mathrm{~mm} \pm 22,6 \mathrm{~mm}$ y la longitud promedio de la AChA de 26,07 $\mathrm{mm} \pm 3,12 \mathrm{~mm}$.

Los orígenes más frecuentes de la AChA se muestran en la Tabla I

Las longitudes de la AChA de ambos lados derecho e izquierdo no mostraron diferencias significativas, así como la distancia de la AChA a la ACoP (Tabla II).

Los calibresde la AChA se muestran en la Tabla III, no existiendo diferencias estadísticamentes significativas entre lados.

Se encontró que no existe correlación $(\mathrm{p}=$ 0,3009) en la longitud de la AChA lado derecho con longitud anteroposterior de la cabeza (Fig. 3 ), en cambio en la AChA del lado izquierdo si se encontró correlación de las variables $(\mathrm{p}=0,0229)$ 


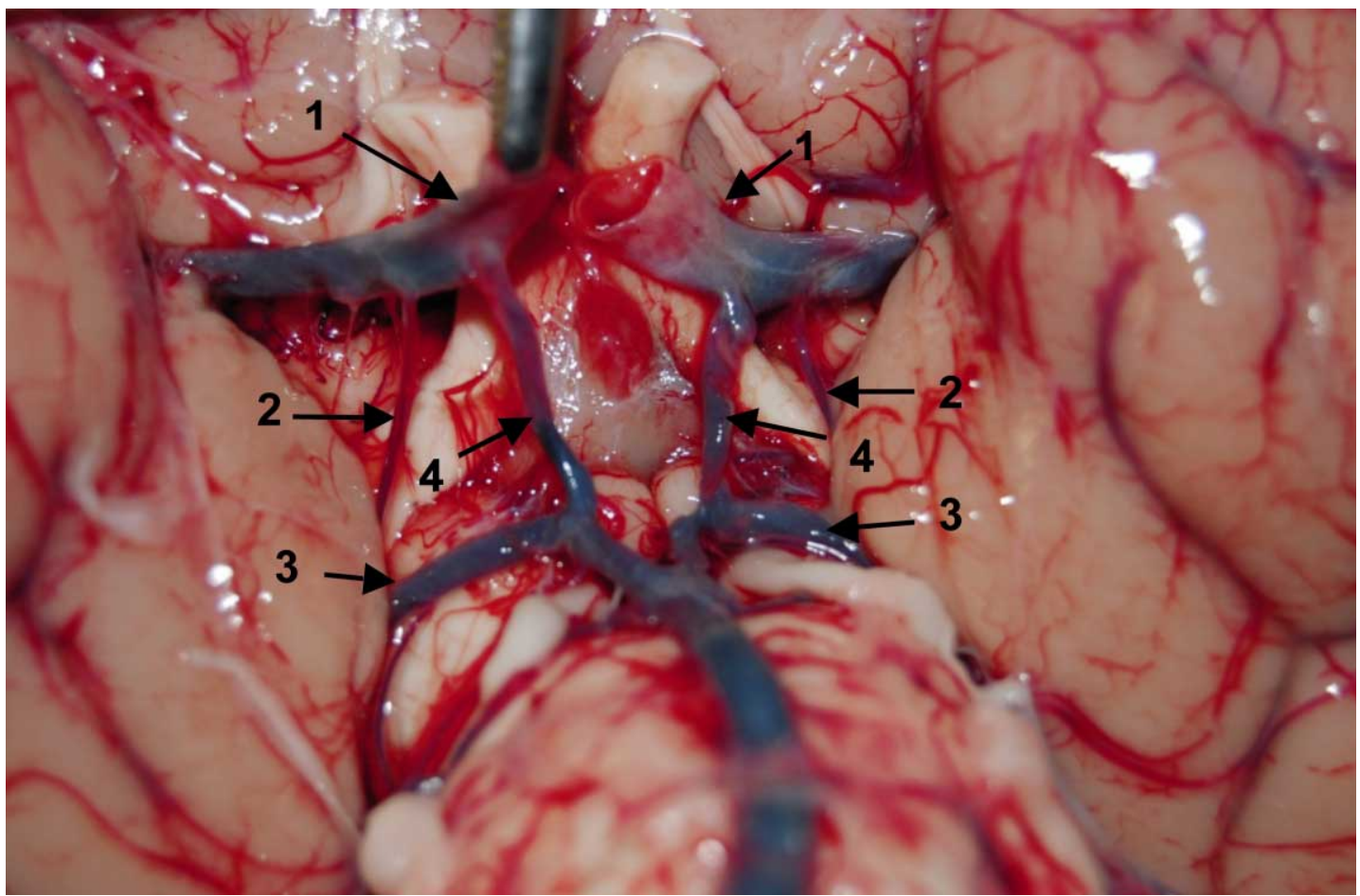

Fig.2. Visión ventral del encéfalo y circulo arterial del cerebro. 1. Arteria carótida interna 2. Arteria coroidea anterior 3. Arteria cerebral posterior. 4. Arteria comunicante posterior.

Tabla I. Orígenes de las AChAs expresados en porcentajes.

\begin{tabular}{lcc}
\hline $\begin{array}{l}\text { Origen de la arteria coroídea } \\
\text { anterior (AChA) }\end{array}$ & Frecuencia & $\%$ \\
\hline A. carótida interna & 26 & 81,25 \\
A. cerebral media & 4 & 12,5 \\
A. comunicante posterior & 2 & 6,25 \\
\hline
\end{tabular}

Tabla II. Valores promedio y desviación estándar de la longitud de la AChA hasta la entrada en su punto plexal y de la distancia al origen de la ACoP.

\begin{tabular}{lrr}
\hline Longitud de AChA & Derecho & Izquierdo \\
\hline Hasta entrada punto plexal & $24,97 \pm 7,92$ & $27,48 \pm 9,93$ \\
Hasta origen ACoP & $3,53 \pm 2,52$ & $2,89 \pm 1,87$ \\
\hline
\end{tabular}

Tabla III. Valores promedio y desviación estándar del calibre de la AChA en distintos puntos de su trayecto.

\begin{tabular}{lcl}
\hline Calibre de AChA & Derecho & Izquier do \\
\hline Origen & $0,73 \pm 0,19$ & $0,70 \pm 0,30$ \\
Tercio medio & $0,66 \pm 0,17$ & $0,57 \pm 0,26$ \\
Entrada punto plexal & $0,52 \pm 0,16$ & $0,52 \pm 0,28$ \\
\hline
\end{tabular}
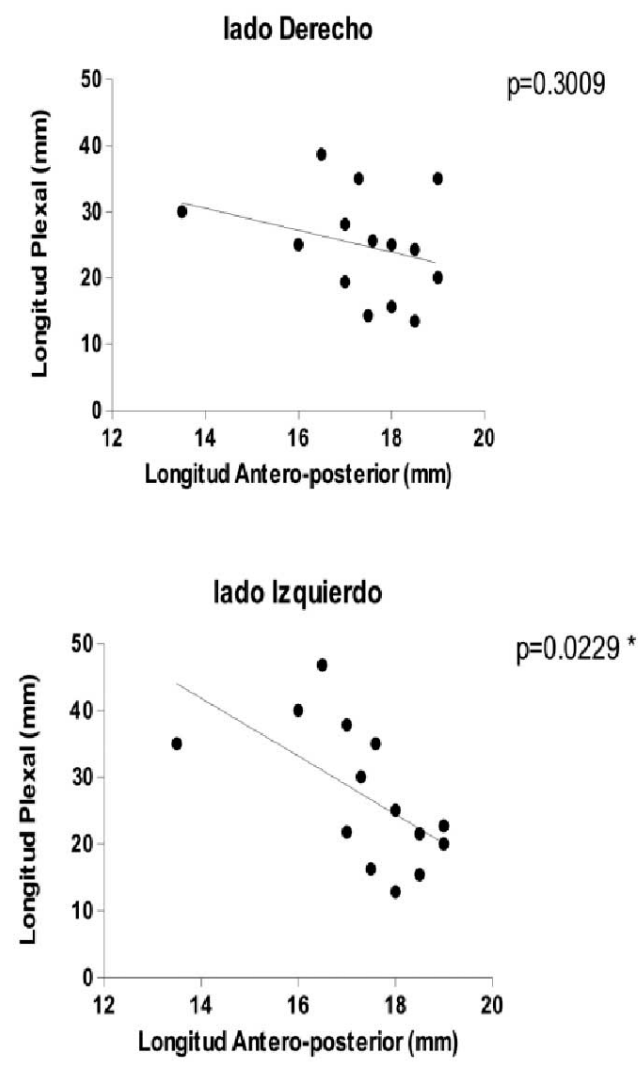

Fig. 3. Correlación de la longitud de la AChA con la longitud antero-posterior de la cabeza en el lado derecho e izquierdo. 
lado Derecho

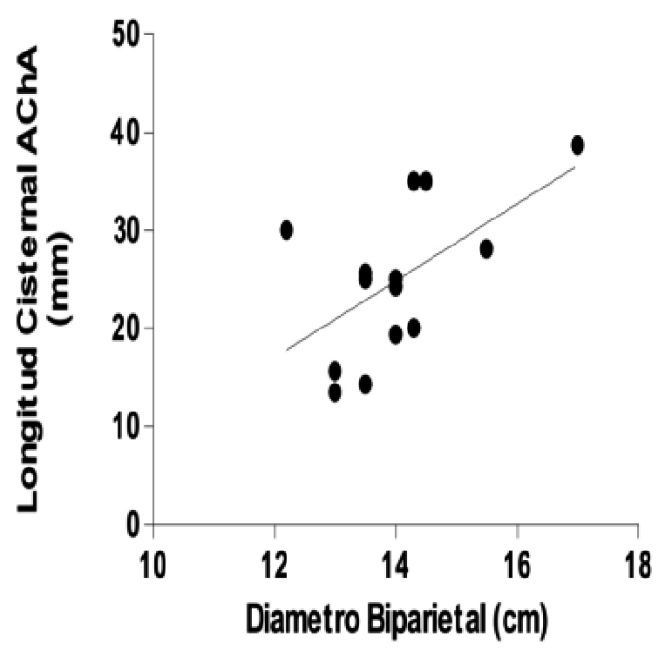

lado lzquierdo

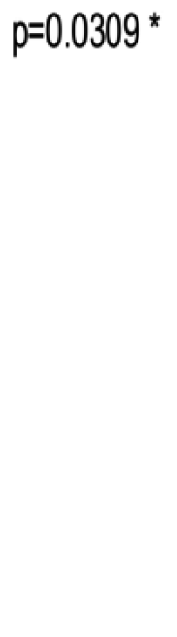

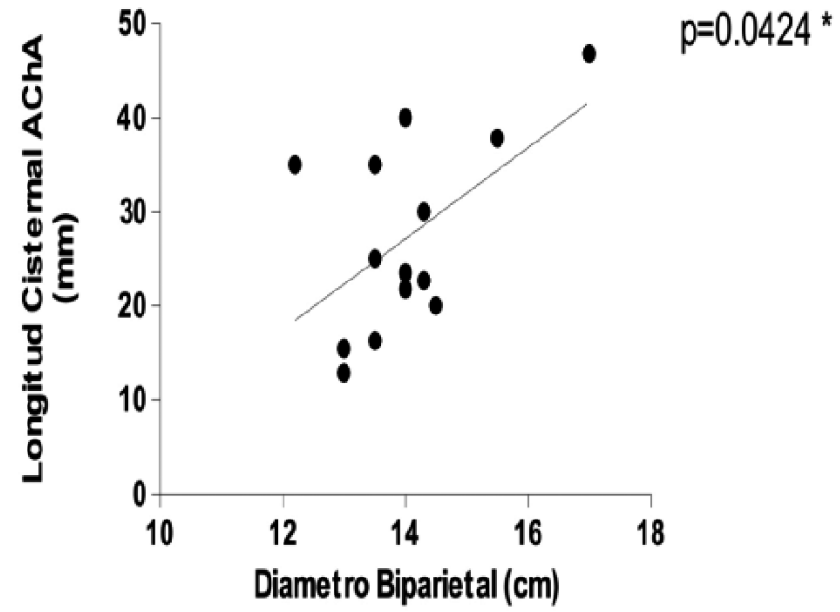

Fig. 4. Correlación del diámetro biparietal con la longitud de la AChA de los lados derecho e izquierdo, existen correlaciones en ambos casos.

\section{DISCUSIÓN}

Nuestras observaciones muestran que el origen más frecuente de la AChA es de la ACI en un 81,25\%, similar a lo señalado por Truek \& Carpenter en el 76,6 \% y de la $\mathrm{ACoP}$ en un $6,25 \%$, agregando origen la ACM en un $12,5 \%$, valores semejantes a los de nuestros análisis.

Por otra parte, Rhoton et al. describen el origen de la AChA en un $98 \%$ de la ACI y en un $2 \%$ de la ACoP, valores que difieren bastante de nuestros resultados, y podrían deberse a diferencias étnicas importantes de considerar. Así, Saeki y Rhoton, encuentran que la AchA se origina en el $100 \%$ de la ACI.

De manera similar, Otomo describe que en menos del $1 \%$ la AChA se originó de la AcoP. Es interesante señalar la diferencia que se plantea en este trabajo, por diversidad racial, realizado en cadáveres de individuos japoneses, estudio que arroja resultados distintos a los obtenidos por Carpenter et al., y Herman et al., en cadáveres caucásicos.

Situación similar ocurre con el calibre registrado por estos autores que es de 1,2 mm. Ferreira \& Braga igualmente destacan cifras similares en relación al origen de la AChA y el promedio de calibre en el segmento cisternal de $0,9 \mathrm{~mm}$ y los de Saeki \& Rhoton con promedio para el calibre de $1,0 \mathrm{~mm}$, variando de $0,5 \mathrm{~mm}$ a 2,3 $\mathrm{mm}$, en comparación a lo obtenido en nuesto estudio, datos menores y corresponden en el tercio medio de la AChA de 0,66 \pm $0,17 \mathrm{~mm}$ en el lado derecho y de $0,57 \pm 0,26 \mathrm{~mm}$ en el izquierdo. En nuestros especímenes no fueron observadas AChA dobles, mencionadas por Saeki \& Rhoton, pero registramos una AChA generada a partir de dos troncos.

Al medir el calibre de la AChA en su origen $(0,73 \mathrm{~mm})$, en el tercio medio $(0,66 \mathrm{~mm})$ y en el punto de entrada al plexo $(0,52 \mathrm{~mm})$ se observa una disminución desde el origen al punto plexal, detalles no mencionados en la literatura consultada. Igualmente, analizando la longitud de la arteria hasta el punto plexalen el lado derecho $(24,97 \mathrm{~mm})$ y lado izquierdo $(27,48 \mathrm{~mm})$, se observan diferencias. La medición de la distancia desde el origen de la AChA al origen de la ACoP en los lados derecho fue de $3,53 \mathrm{~mm}$ e izquierdo de $2,89 \mathrm{~mm}$, no observándose diferencias significativas entre los autores citados.

En trabajos previos (Mandiola et al. 2006, 2010) sobre las arterias comunicantes del círculo arterial del cerebro, se procuró establecer la correlación existente entre estas arterias y mediciones antropológicas cefálicas, correlaciones que también se analizan en este trabajo, utilizando para ello los datos obtenidos de la AChA.

De esta manera, se observó correlación entre el diámetro biparietal y la longitud cisternal de la AChA, hallazgo que parece interesante resaltar. En trabajos de Fujimoto \& Tanaka, Goldberg, González, Kamath, Krupa \& Krupa, Lazorthes et al., Marinkovic et al., Orlandini et al., entre otros, especificaron datos de la observación de vasos arteriales encefálicos, no habiendo mención de correlacio- 
nes biométricas de los componentes del círculo arterial del cerebro con características antropológicas de los especímenes estudiados.

Marinkovic et al. estudiaron las ramas perforantes de la AChA y establecieron que su número tiene un rango entre 2 y 9 , con promedio de 4,6 siendo diferente a lo observado en nuestra investigación, donde tuvimos rangos entre 4 a 6 ramas en el lado derecho y entre 3 a 4 en el lado izquierdo. Sin embargo, hay autores como Milisavljevic et al. y Servet et al., quienes afirmaron que las variaciones en el calibre de los vasos, incluyendo otros factores como edad y sexo, sumados a factores genéticos son importantes en la conformación de padrones morfológicos.

La arteria coroídea anterior presenta variabilidad, cuando se considera el lado, y se observa su relación con detalles bio-antropológicos cefálicos, por tanto es difícil fijar puntos de reparo para trayectos vasculares

Los datos biométricos aportados son importantes y deberían constituir una base de análisis a considerar.

MANDIOLA, E.; ALARCÓN, E.; MONTERO, C.; PULLEGHINI, M.; DEL SOL, M.; OLAVE, E. \& OÑATE, J. C. Biometrical characteristics of anterior choroidal artery in its cisternal segment. Int. J. Morphol., 30(3):1050-1055, 2012.

SUMMARY:The clinical implications of occlusive processes in the anterior choroidal artery (AChA) are of considerable importance and have motivated us to perform a biometrical analysis of the afore mentioned artery including new biometric parameters (measurements). This study was perfomed on vascularized brains that were both fixed with for formalin solution and injected whith red tinted latex and brains that were not fixed with formalin solution, which were obtained from the Medical Legal Service, Santiago. They were observed using a luminescent $4 \mathrm{X}$ magnifying glass and the measurements perfomed using a Mitutoyo Digital Caliper. This artery originated from the internal carotid artery (ICA) in $83.3 \%$ of the cases observed, and in $16.7 \%$ it originated from the posterior communicating artery ( PCoA). The mean caliber (diameter) of the artery was $0.68 \pm 0.31 \mathrm{~mm}$ at the point of origin; the middle third was $0.60 \mathrm{~mm} \pm 0.21 \mathrm{~mm}$; at the point of entry into the choroidal plexus it was $0.48 \mathrm{~mm} \pm 0.23 \mathrm{~mm}$. The mean length from its origin at the AChA to the choroidal plexus was $28.67 \mathrm{~mm} \pm 6.33 \mathrm{~mm}$; the mean distance from its origin at the PCoAwas $6.97 \mathrm{~mm} \pm 4.89 \mathrm{~mm}$. The number of perforating branches of the AChA was 6 . Our results can be considered a clinical contribution.

KEY WORDS : Anterior choroidal artery ; Biometry; Cephalix index; Internal carotid artery.

\section{REFERENCIAS BIBLIOGRÁFICAS}

Adachi, B. Das arteriensystem der japaner. Kyoto, Marusen Co., 1928.

Ferreira, A. \& Braga, F. M.Microsurgical of the anterior choroidal artery. Arq. Neuropsiquiatr. 48(4):448-53, 1990.

Fujimoto, K. \& Tanaka, O. Morphological examination of the circulus arteriosus cerebri humani (Circle of Willis). Anterior and posterior communicating arteries Kaibogaku Zasshi, 64 (5):481-9, 1989.

Goldberg, H. I.The anterior choroidal artery. In Newton, T. H.,Potts, D.G. Radiology of the Skull and Brain. St. Louis, Mosby, 1974 .Vol. II. pp.1628-58.

González, A. Círculo arterial de Willis. Sus medidas externas y variaciones anatómicas. Acta Neurol. Latinoamer., 5:1-6, 1959.

Herman, L. H.; Fernando, O. V. \& Gurdjian, E. S. The anterior choroidalartery : an anatomical study of its area of distribution. Anat. Rec., 154:95-102, 1966.

Krupa, B. \& Krupa, U. Variations of the anterior communicating artery. Folia Morphol., 57(3):223-40, 1998.
Lazorthes, G.; Gouaze, A; Santini, J-J \& Salamon, G. Le circle arteriel du cerveau (circulus arteriosus cerebri). Anat. Clinica, 1:241-57, 1979.

Mandiola, E. Alarcón, E; Oñate, J. C.; del Sol, M. \& Olave, E. Arterias comunicantes del circulo arterial del cerebro: Características biométricas y relación con el índice cefálico. Int. J. Morphol., 28(3): 957-61, 2010.

Mandiola, E.; Alarcón, E; Oñate, J.C.; Sanhueza, P.; del Sol, M. \& Olave, E. Biometría de las arterias comunicantes posteriores y anteriores en su segmento precomunicantes (P1) en el circulo arterial del cerebro (Willis). Int. J. Morphol., 24(4):601-6, 2006.

Marinkovic, S.; Gibo, H.; Brigante, L.; Nikodijevic, I \& Petrovic, P. The surgical anatomy of the perforating branches of the anterior choroidal artery. Surg. Neurol., 52:30-6, 1999.

Martin, R. \& Saller, K. Lehrbuch der Antthropologie. Sttugart, Fischer Verlag.1957. Abt. 1.

Milisavljevic, M.; Marinkovic, S.; Lolic-Draganic, V. \& Djordjevic, L. Anastomoses in the territory of the posterior cerebral arteries. Acta Anat., 127:122-5, 1986. 
Orlandini, G.; Ruggiero, C.; Orlandini, S. Z. \& Gulisano, M. Blood vessel size of circulus arteriosus cerebri (circle of Willis): A statistical Research on 100 Human Subjects. Acta Anat., 123:72-6, 1985.

Orts Llorca, F. Anatomía humana. $3^{\text {a }}$. Ed. Barcelona, Científico Médica, 1964. V.2.

Otomo, E. The anterior choroidal artery. Arch. Neurol., 13: 656-8, 1965.

Rhoton, A. L. Jr; Fuji, K. \& Fradd, B. Microsurgical anatomia of the anterior choroidal artery. Surg. Neurol., 12(2):171-87, 1979.

Rosner, S.;Rhoton, A.;Ono, M. \& Barry, M. Microsurgical anatomy of the anterior perforating arteries. J. Neurosurg., 61:468- 85, 1984.

Rouviere, H. \& Delmas, A. Anatomía Humana, Descriptiva, Topográfica y Funcional.11 ${ }^{\mathrm{a}}$ Ed. Barcelona, Masson, 2005. V. 4.

Saeki, N. \& Rhoton, A.Microsurgical anatomy of the upper basilar artery and the posterior circle of Willis. J. Neurosurg., 46:56378, 1977.

Sengupta, R.P. Anatomical variations in the origin of teh posterior cerebral artery demostred by carotid angiography and their significance in the direct surgical treatment of posterior communicating aneurysms. Neurochirugia, 18(2):33-42, 1975.

Servet, I.; Anil, A. \& Tuncalp, O. Distal anterior choroidal artery aneurysms. Surg. Neurol., 67:46-52, 2007.

Testut, L. \& Latarjet, A. Tratado de Anatomía Humana. $9^{\mathrm{a}}$ Ed. Salvat, Barcelona, 1964 .V. 2.

Truex, R. \& Carpenter, M. Neuroanatomía Humana. $4^{\mathrm{a}}$.ed. Buenos Aires, Ateneo, 1973.

Wackenheim, A.; Ludwicza, R. \& Capesius, P. The course of the anterior choroidal artery. Neuroradiology, 11:73-5, 1976.

Warwick, R. \& Williams, P. Gray Anatomia. 35 ${ }^{\text {a }}$ Ed. Rio de Janeiro. Guanabara Koogan, 1979 . V. 2.
Dirección para correspondencia:

Prof. Dr. Eduardo Mandiola

Facultad de Medicina

Departamento de Morfología

Universidad Andrés Bello

República 275. Piso 2.

Santiago

CHILE

Email: emandiola@unab.cl

Recibido : 15-03- 2012

Aceptado : 24-05-2012 